\title{
ON THE NONEXISTENCE OF $L^{p}$ SOLUTIONS OF CERTAIN NONLINEAR DIFFERENTIAL EQUATIONS
}

\author{
by THOMAS G. HALLAM
}

(Received 9 December, 1966)

The asymptotic behavior of the solutions of ordinary nonlinear differential equations will be considered here. The growth of the solutions of a differential equation will be discussed by establishing criteria to determine when the differential equation does not possess a solution that is an element of the space $L^{p}(0, \infty)(p \geqq 1)$.

The first theorem below gives a sufficient condition which guarantees that the solutions of a certain differential equation are not in $L^{p}(0, \infty)$. This theorem is an extension of a result originally due to Wintner [8], where a second-order linear differential equation was considered. This result was successfuly extended to nonlinear second-order differential equations by Suyemoto and Waltman [6] and Burlak [2]. Our extension is to an $n$th order nonlinear differential equation, namely,

$$
y^{(n)}+g(t, y)=0, \quad y^{(n)}=\frac{d^{n} y}{d t^{n}}
$$

where

$$
|g(t, y)| \leqq f(t)|y|^{r}
$$

with $n \geqq 1, r \geqq 1$, and $f(t)$ continuous on $[0, \infty)$.

We shall assume throughout that $g(t, y)$ is sufficiently smooth to guarantee the existence of solutions of (1). The word "solution", for the remainder of this note, will mean a nontrivial (i.e., not identically zero) solution that exists on the interval $[0, \infty)$.

THEOREM 1. Let $y(t)$ be any solution of equation (1) with condition (2) imposed; then $y(t)$ is not in $L^{2 r}(0, \infty)$ provided that

$$
\int_{0}^{\infty} t^{2 n-1} f^{2}(t) d t<\infty
$$

Proof. The proof is similar to that given in the above references and requires the following propositions.

(I) If equation (1) has a solution $y(t)$ in $L^{2 r}(0, \infty)$ and (3) is satisfied, then $y^{(n-1)}(t)$, $y^{(n-2)}(t), \ldots, y^{(1)}(t), y(t)$ approach zero as $t$ approaches infinity.

(II) If equation (1) has a solution $y(t)$ in $L^{2 r}(0, \infty)$ and (3) is satisfied, then $y(t)$ is in $L^{2}(0, \infty)$.

Proposition (I) will now be established. From equation (1), we obtain

$$
y^{(n-1)}(t)-y^{(n-1)}(0)=-\int_{0}^{t} g(s, y(s)) d s .
$$


Considering the integral in equation (4), using inequality (2) and the Schwarz Inequality, we obtain

$$
\begin{aligned}
\left|\int_{0}^{t} g(s, y(s)) d s\right| & \leqq \int_{0}^{t}|g(s, y(s))| d s \\
& \leqq \int_{0}^{t} f(s)|y(s)|^{r} d s \\
& \leqq\left[\int_{0}^{t} f^{2}(s) d s\right]^{\frac{1}{2}}\left[\int_{0}^{t}|y(s)|^{2 r} d s\right]^{\frac{1}{2}} .
\end{aligned}
$$

If $y(t)$ is a solution of $(1)$ in $L^{2 r}(0, \infty)$, then, from (4), it follows that $\lim _{t \rightarrow \infty} y^{(n-1)}(t)$ exists, since the integral is majorized by

$$
\left[\int_{0}^{\infty} f^{2}(s) d s\right]^{\frac{1}{2}}\left[\int_{0}^{\infty}|y(s)|^{2 r} d s\right]^{\frac{1}{2}}
$$

If $\lim _{t \rightarrow \infty} y^{(n-1)}(t)=A_{n-1} \neq 0$, then $\int_{0}^{\infty}|y(t)|^{2 r} d t$ diverges, which contradicts the hypothesis of proposition (I); thus $A_{n-1}=0$.

Suppose that it has been established that $\lim _{t \rightarrow \infty} y^{(n-j)}(t)=0$, where $j$ is some fixed integer such that $1 \leqq j \leqq n-1$. From equation (1), we obtain

$$
y^{(n-j)}(t)=(-1)^{j+1} \int_{t}^{\infty} \int_{t_{1}}^{\infty} \ldots \int_{t_{j-1}}^{\infty} g\left(t_{j}, y\left(t_{j}\right)\right) d t_{j} d t_{j-1} \ldots d t_{1} .
$$

Integration of the above equation, inequality (2) and the Schwarz Inequality lead to the inequality

$$
\begin{aligned}
\int_{0}^{\infty}\left|y^{(n-j)}(t)\right| d t & \leqq \int_{0}^{\infty} \int_{t}^{\infty} \int_{t_{1}}^{\infty} \ldots \int_{t_{j-1}}^{\infty}\left|g\left(t_{j}, y\left(t_{j}\right)\right)\right| d t_{j} d t_{j-1} \ldots d t_{1} d t \\
& \leqq \int_{0}^{\infty} t_{j}^{j}\left|g\left(t_{j}, y\left(t_{j}\right)\right)\right| d t_{j} \\
& \leqq \int_{0}^{\infty} s^{j} f(s)|y(s)|^{r} d s \\
& \leqq\left[\int_{0}^{\infty} s^{2 j} f^{2}(s) d s\right]^{\frac{1}{2}}\left[\int_{0}^{\infty}|y(s)|^{2 r} d s\right]^{\frac{1}{2}} .
\end{aligned}
$$

The last integrals in (5) are finite by hypothesis. Therefore, since $\int_{0}^{\infty}\left|y^{(n-j)}(s)\right| d s$ converges, $\lim _{t \rightarrow \infty} y^{(n-j-1)}(t)$ exists; let this limit be $A_{n-j-1}$. Again, under the hypothesis of $(\mathrm{I})$, it is necessary that $A_{n-j-1}=0$. This establishes proposition (I). 
To show that (II) holds, let $y(t)$ be a solution of (1) in $L^{2 r}(0, \infty)$. Using (I), we may write

$$
y(t)=(-1)^{n-1} \int_{t}^{\infty} \int_{t_{1}}^{\infty} \ldots \int_{t_{n-2}}^{\infty} \int_{t_{n-1}}^{\infty} g\left(t_{n}, y\left(t_{n}\right)\right) d t_{n} d t_{n-1} \ldots d t_{1} .
$$

Proceeding as in (5) above, we obtain the inequality

$$
|y(t)| \leqq \int_{t}^{\infty} \int_{t_{1}}^{\infty} \ldots \int_{t_{n-2}}^{\infty} \int_{t_{n-1}}^{\infty} f\left(t_{n}\right)\left|y\left(t_{n}\right)\right|^{r} d t_{n} d t_{n-1} \ldots d t_{1} .
$$

Therefore

$$
\begin{aligned}
\int_{0}^{\infty}|y(t)|^{2} d t & \leqq \int_{0}^{\infty}\left[\int_{t}^{\infty} \int_{t_{1}}^{\infty} \ldots \int_{t_{n-2}}^{\infty} \int_{t_{n-1}}^{\infty} f\left(t_{n}\right)\left|y\left(t_{n}\right)\right|^{r} d t_{n} d t_{n-1} \ldots d t_{1}\right]^{2} d t \\
& \leqq \int_{0}^{\infty}\left[\int_{t}^{\infty} t_{n}^{2(n-1)} f^{2}\left(t_{n}\right) d t_{n}\right]\left[\int_{t}^{\infty}\left|y\left(t_{n}\right)\right|^{2 r} d t_{n}\right] d t \\
& \leqq\left[\int_{0}^{\infty}|y(s)|^{2 r} d s\right]\left[\int_{0}^{\infty} s^{2 n-1} f^{2}(s) d s\right]<\infty .
\end{aligned}
$$

The above inequality shows that the existence of a solution $y(t)$ in $L^{2 r}(0, \infty)$ implies that $y(t)$ is in $L^{2}(0, \infty)$; that is, proposition (II) is verified.

To complete the proof of Theorem 1, suppose that there exists a solution $y(t)$ of (1) which is in $L^{2 r}(0, \infty)$. By virtue of $(\mathrm{I}), \lim _{t \rightarrow \infty} y(t)=0$; therefore there exists a $t=T$ such that, for all $t \geqq T,|y(t)|<1$, and $y(t) \neq 0$ on $[T, \infty)$. For $r \geqq 1$ and $t \geqq T$, we have $|y(t)|^{2 r} \leqq|y(t)|^{2}$ and

$$
\int_{1}^{\infty}|y(t)|^{2 r} d t \leqq \int_{t}^{\infty}|y(t)|^{2} d t
$$

An argument similar to that used in (6) with $t \geqq T$ leads to

Since

$$
\begin{aligned}
\int_{t}^{\infty}|y(s)|^{2} d s & \leqq\left[\int_{t}^{\infty}|y(s)|^{2 r} d s\right]\left[\int_{t}^{\infty} s^{2 n-1} f^{2}(s) d s\right] \\
& \leqq\left[\int_{t}^{\infty}|y(s)|^{2} d s\right]\left[\int_{t}^{\infty} s^{2 n-1} f^{2}(s) d s\right] .
\end{aligned}
$$

from (7) we have

$$
\int_{t}^{\infty}|y(s)|^{2} d s>0
$$

$$
1 \leqq \int_{t}^{\infty} s^{2 n-1} f^{2}(s) d s
$$

However, this contradicts (3) and concludes the proof of the theorem. 
Remark 1. It is clear that the above theorem can be extended to a differential equation of the type

where $g$ satisfies

$$
y^{(n)}+g\left(t, y, y^{(1)}, y^{(2)}, \ldots, y^{(n-1)}\right)=0,
$$

$$
\left|g\left(t, y, y^{(1)}, y^{(2)}, \ldots, y^{(n-1)}\right)\right| \leqq f(t)|y(t)|^{r} ;
$$

that is, $g$ is a bounded function of $y^{(1)}, y^{(2)}, \ldots, y^{(n-1)}$. The condition (3) is, in a sense, the best possible, as it cannot be replaced by

$$
\int_{0}^{\infty} t^{2 n-1+\varepsilon} f^{2}(t) d t<\infty \text { and } \int_{0}^{\infty} t^{2 n-1} f^{2+\varepsilon}(t) d t<\infty
$$

with $\varepsilon>0$ (see [8]).

For the remainder of this note we shall be primarily concerned with obtaining a sufficient condition for some of the solutions of a nonoscillatory differential equation not to be in $L^{p}(0, \infty)(p \geqq 1)$. In [4] Kurss used the Sturm Comparison Theorems in an eigenvalue problem to determine the nonexistence of solutions of a linear differential equation in the Hilbert space $L^{2}(0, \infty)$. In what follows we shall use a nonlinear analogue of the Sturm Comparison Theorem due to Sevelo and Stelik [5] to establish the nonexistence of $\left(L^{p}\right)$ solutions of a nonlinear second-order differential equation (cf. Theorem 4 below). Also, we shall give a numerical comparison theorem for a pair of nonlinear differential equations (cf. Theorem 3 below).

Consider the differential equations

$$
\begin{gathered}
{\left[p(t) u^{\prime}\right]^{\prime}+f_{1}\left(t, u, u^{\prime}\right)=0,} \\
{\left[p(t) v^{\prime}\right]^{\prime}+f_{2}\left(t, v, v^{\prime}\right)=0,}
\end{gathered}
$$

where $p^{\prime}(t)$ is continuous on $[0, \infty), p(t) \geqq p_{0}>0, p_{0}$ is constant, and the $f_{1}\left(t, z, z^{\prime}\right)(i=1,2)$ are continuous and satisfy conditions sufficient to guarantee the existence and uniqueness of solutions of (8) and (9) for $t \in[0, \infty)$.

The following result can be found in [5].

THEOREM 2. If $f_{2}\left(t, v, v^{\prime}\right) / v-f_{1}\left(t, u, u^{\prime}\right) / u \geqq 0$ for all $u^{\prime}, v^{\prime}, t \in[0, \infty), u \in U=(-\bar{u}, \bar{u})$, $v \in V=(-\bar{v}, \bar{v})(0<\bar{u} \leqq \infty, 0<\bar{v} \leqq \infty)$, then between any two consecutive zeros of an arbitrary solution $u(t) \in U$ of equation (8) there is situated at least one zero of each solution $v(t) \in V$ of equation (9).

Remark 2. Subject to the hypotheses of the above theorem, we observe that, if equation (9) has a nonoscillatory solution $v(t)$ in $V$, then all solutions $u(t)$ in $U$ of equation (8) are nonoscillatory. This is true because, if some solution of $(8)$ is oscillatory, then, by Theorem 2 , all solutions of (9) are oscillatory.

THEOREM 3. Let $u(t)$ and $v(t)$ be solutions of (7) and (8), respectively, such that

$$
u\left(t_{0}\right)=v\left(t_{0}\right) \neq 0, \quad u^{\prime}\left(t_{0}\right)=v^{\prime}\left(t_{0}\right) .
$$


If

$$
f_{2}\left(t, v, v^{\prime}\right) / v>f_{1}\left(t, u, u^{\prime}\right) / u
$$

for all $t \in\left[t_{0}, \infty\right), u^{\prime}, v^{\prime}, u, v$, where $0<|v| \leqq|u|$, then $|u(t)|>|v(t)|$ provided that $v(t)$ is not zero. identity

Proof. The proof follows that given by Tricomi $[7$, p. 103] and is a consequence of the

$$
p(t)\left[v u^{\prime}-u v^{\prime}\right](t)=\int_{t_{0}}^{t} u(s) v(s)\left[\frac{f_{2}\left(s, v(s), v^{\prime}(s)\right)}{v(s)}-\frac{f_{1}\left(s, u(s), u^{\prime}(s)\right)}{u(s)}\right] d s .
$$

THEOREM 4. Let inequality (10) hold for all $t \in[0, \infty), u^{\prime}, v^{\prime}, u$, and $v$; furthermore, suppose that equation (9) is nonoscillatory. If some solution of (9) is not in $L^{p}(0, \infty)(p \geqq 1)$, then some solution of $(8)$ is not in $L^{p}(0, \infty)$.

Proof. If $v(t)$ is a solution of the nonoscillatory equation (9) which is not in $L^{p}(0, \infty)$, then, by Remark 2 with $f_{1}=f_{2}, v(t)$ is nonoscillatory. Hence there exists a $t=t_{0}$ such that $|v(t)|>0$ for $t \geqq t_{0}$. By virtue of Theorem 3, it follows that the solution $u(t)$ of equation (8) that satisfies the initial conditions

also satisfies the inequality

$$
u\left(t_{0}\right)=v\left(t_{0}\right), \quad u^{\prime}\left(t_{0}\right)=v^{\prime}\left(t_{0}\right)
$$

$$
|u(t)|>|v(t)| \text {. }
$$

Therefore $u(t)$ is not in $L^{p}\left(t_{0}, \infty\right)$ and consequently not in $L^{p}(0, \infty)$.

Remark 3. The above theorem can be used as an $\left(L^{p}\right)$-existence theorem for equation (9). We illustrate this by the following result of Bellman [1].

If all the solutions of

$$
u^{\prime \prime}+a(t) u=0
$$

belong to $L^{2}(0, \infty)$, then all the solutions of

$$
v^{\prime \prime}+\{a(t)+b(t)\} v=0
$$

belong to $L^{2}(0, \infty)$ provided that $|b(t)| \leqq c_{1}(t>0)$.

It follows from Theorem 4 that the same conclusion is now obtained without the boundedness condition $|b(t)| \leqq c_{1}(t>0)$, but under the different condition that (12) is nonoscillatory. Actually, the equation (12) may be generalized to a nonlinear equation; for example,

$$
v^{\prime \prime}+a(t) v+\sum_{j=1}^{n} b_{j}(t) v^{2 j-1}=0,
$$

where $b_{j}(t) \geqq 0$ for all $j=1,2, \ldots, n$, and $b_{k}(t)>0$ for some $k=1,2, \ldots, n$.

However, even for the linear case, the above result is not easy to apply. To observe this, we consider the following question: When does the nonoscillatory linear equation (11) 
possess all solutions in $L^{2}(0, \infty)$ ? (The fact that (11)-and not merely (12)-must be nonoscillatory results from Remark 2 above.)

As a partial answer, we observe that $a(t)$ cannot be bounded [1, p. 138, Problem 8]. Also, $a(t)$ cannot be of constant sign; for if $a(t)<0$ on $[0, \infty)$, consider the equations $v^{\prime \prime}=0$ and $u^{\prime \prime}+a(t) u=0$. The hypotheses of Theorem 4 are satisfied and, consequently, all solutions of $u^{\prime \prime}+a(t) u=0$ cannot be in $L^{2}(0, \infty)$. On the other hand, if $a(t)>0$ on $[0, \infty), \int^{\infty} a(t) d t<\infty$ is necessary for nonoscillation [3, p. 367]; and, as remarked above, $a(t)$ cannot be bounded. However, if $a(t)>0$, then all solutions of (11) are either oscillatory or monotone; hence, in the case under consideration, all solutions must be monotone. But, if $\int^{\infty} a(t) d t<\infty$, then all solutions cannot be bounded [1, p. 121, Problem 2]; therefore all solutions cannot be in $L^{2}(0, \infty)$.

Thus, if equation (11) is to be nonoscillatory and have all solutions in $L^{2}(0, \infty)$, then it is necessary that $a(t)$ be oscillatory and $\limsup _{t \rightarrow \infty}|a(t)|=\infty$. In this situation it is an open question if the hypotheses are compatible with those on $f_{1}$ and $f_{2}$ in Theorems 2, 3 and 4.

\section{REFERENCES}

1. R. Bellman, Stability theory of differential equations, McGraw-Hill (New York, 1953), p. 116.

2. J. Burlak, On the nonexistence of $L^{\mathrm{a}}$ solutions of a class of nonlinear differential equations, Proc. Edinburgh Math. Soc. 14 (1965), 257-268.

3. P. Hartman, Ordinary differential equations, Wiley (New York, 1964).

4. H. Kurss, A limit point criterion for nonoscillatory Sturm-Liouville differential operators; to appear.

5. V. N. Ševelo and V. G. Štelik, Certain problems concerning the oscillation of solutions of nonlinear nonautonomous second order equations, Soviet Math. Dokl. 4 (1963), 383-387.

6. L. Suyemoto and P. Waltman, Extension of a theorem of A. Wintner, Proc. Amer. Math. Soc. 14 (1963), 970-971.

7. F. G. Tricomi, Differential equations, Hafner (New York, 1961).

8. A. Wintner, A criterion for the nonexistence of $\left(L^{2}\right)$-solutions of a nonoscillatory differential equation, J. London Math. Soc. 25 (1950), 347-351.

FLORIDA STATE UNIVERSITY

Tallahassee, Florida 